\title{
NGC 6139: a normal massive globular cluster, or a first-generation dominated cluster? Clues from the light elements ${ }^{\star \star \star \star, \star \star \star}$
}

\author{
A. Bragaglia ${ }^{1}$, E. Carretta ${ }^{1}$, A. Sollima ${ }^{1}$, P. Donati ${ }^{1,2}$, V. D'Orazi ${ }^{3,4,5}$, R. G. Gratton ${ }^{3}$, S. Lucatello ${ }^{3}$, and C. Sneden ${ }^{6}$ \\ 1 INAF-Osservatorio Astronomico di Bologna, via Ranzani 1, 40127 Bologna, Italy \\ e-mail: angela.bragaglia@oabo.inaf.it \\ 2 Dipartimento di Fisica e Astronomia, Università di Bologna, viale Berti Pichat 6, 40127 Bologna, Italy \\ 3 INAF-Osservatorio Astronomico di Padova, Vicolo dell'Osservatorio 5, 35122 Padova, Italy \\ ${ }^{4}$ Monash Centre for Astrophysics, School of Physics and Astronomy, Monash University, Melbourne, VIC 3800, Australia \\ 5 Department of Physics and Astronomy, Macquarie University, Sydney, NSW 2109, Australia \\ ${ }^{6}$ Department of Astronomy and McDonald Observatory, The University of Texas, Austin, TX 78712, USA
}

Received 25 May 2015 / Accepted 30 July 2015

\section{ABSTRACT}

\begin{abstract}
Information on globular clusters (GC) formation mechanisms can be gathered by studying the chemical signature of the multiple populations that compose these stellar systems. In particular, we investigate the anti-correlations among $\mathrm{O}, \mathrm{Na}, \mathrm{Al}$, and $\mathrm{Mg}$ to explore the influence of cluster mass and environment on GCs in the Milky Way and in extragalactic systems. We present here the results obtained on NGC 6139, which, on the basis of its horizontal branch morphology, has been proposed to be dominated by first-generation stars. In our extensive study based on high-resolution spectroscopy, the first for this cluster, we found a metallicity of $[\mathrm{Fe} / \mathrm{H}]=$ $-1.579 \pm 0.015 \pm 0.058$ ( $\mathrm{rms}=0.040 \mathrm{dex}, 45$ bona fide member stars) on the UVES scale defined by our group. The stars in NGC 6139 show a chemical pattern normal for GCs, with a rather extended $\mathrm{Na}-\mathrm{O}$ (and Mg-Al) anti correlation. NGC 6139 behaves as expected from its mass and contains a large portion (about two thirds) of second-generation stars.
\end{abstract}

Key words. stars: abundances - stars: atmospheres - stars: Population II - Galaxy: general - globular clusters: general globular clusters: individual: NGC 6139

\section{Introduction}

Once considered as a good example of simple stellar populations, Galactic globular clusters (GCs) are currently thought to have formed in a complex chain of events, which left a fossil record in their chemical composition (see e.g., the review by Gratton et al. 2012). Photometrically, GCs often exhibit spread, split, and even multiple sequences that can be explained by different chemical composition among cluster stars, in particular of light elements such as $\mathrm{He}, \mathrm{C}, \mathrm{N}$, and $\mathrm{O}$ (e.g., Carretta et al. $2011 \mathrm{~b}$; Sbordone et al. 2011; Milone et al. 2012a; Piotto et al. 2015). Our FLAMES survey of more than 20 Milky Way (MW) GCs (see Carretta et al. 2009a, 2014b, and references therein) combined with literature data, demonstrated that most, perhaps all, GCs host multiple stellar populations (see Carretta et al. 2010a). Variations of $\mathrm{Na}$ and $\mathrm{O}$ (and sometimes of $\mathrm{Mg}$ and $\mathrm{Al}$ ) abundances trace these different sub-populations.

Our large and homogeneous database allowed us a quantitative study of the $\mathrm{Na}-\mathrm{O}$ anti-correlation. In all the analysed GCs we found about one third of stars to be of primordial composition, similar to that of field stars of similar metallicity (low

\footnotetext{
* Based on observations collected at ESO telescopes under programme 093.B-0583 and on data obtained from the ESO Science Archive Facility under request number 94403.

$\star \star$ Appendix $\mathrm{A}$ is available in electronic form at http: //www . aanda.org

$\star \star \star$ The final photometric catalogue is only available at the CDS via anonymous ftp to cdsarc.u-strasbg. fr (130.79.128.5) or via http://cdsarc.u-strasbg.fr/viz-bin/qcat?]/A+A/583/A69
}

$\mathrm{Na}$, high O), which means that they belong to the first generation (FG). The other two thirds have a modified composition (increased $\mathrm{Na}$, depleted $\mathrm{O}$ ) and belong to the second generation (SG) of stars, which is polluted by the FG (see, e.g., Carretta et al. 2009a,b). It is still debated which were the more massive stars of the FG that produced the gas of modified composition, see for instance Decressin et al. (2007), Ventura et al. (2001), or Bastian et al. (2013) for an alternative view.

We also found that the extension of the $\mathrm{Na}-\mathrm{O}$ anti-correlation tends to be larger for higher mass GCs and that, apparently, there is an observed minimum cluster mass for the appearance of the Na-O anti-correlation (Carretta et al. 2010a). It is important to understand whether this limit is due to the small statistics (fewer low-mass clusters have been studied, and only a few stars were observed in each). This in an important constraint for cluster formation mechanisms because it indicates the mass at which we expect that a cluster is able to retain part of the ejecta of the FG, hence to show the $\mathrm{Na}-\mathrm{O}$ signature (the masses of the original clusters are expected to be much higher than the present ones, since the SG has to be formed by the ejecta of the FG).

Variations in $\mathrm{Na}, \mathrm{O}$, and $\mathrm{He}$ are connected with each other, but do not tell us exactly the same story. The effects of increased $\mathrm{He}$ are visible in colour-magnitude diagrams (CMD) in the main-sequence (MS) phase (e.g., Piotto et al. 2007) or, more evidently, in the horizontal branch phase (HB, e.g., D'Antona et al. 2005; Gratton et al. 2010; Milone et al. 2014). Based on the possibility of reproducing their HBs with a single He value, Caloi \& D'Antona (2011) proposed that some GCs are composed of a single generation (FG-only) or predominantly by a 
single generation (mainly-FG). It would be important to measure their $\mathrm{Na}, \mathrm{O}$, and $\mathrm{Al}$ abundances to clarify the issue. Al, which is only produced at higher temperatures than $\mathrm{Na}$ (e.g., Prantzos et al. 2007, for an application to the GC peculiar chemistry), that is, by more massive stars, should follow the He enrichment better than $\mathrm{Na}$ (and $\mathrm{O}$ ). We can then expect to find $\mathrm{Na}$ and $\mathrm{O}$ variations without significant $\mathrm{He}$ enhancement; this would produce short HBs (and no variations in $\mathrm{Al})^{1}$. In summary, we would be in presence of FG and SG stars even without significant He variations; this is important to understand the cluster formation and early intracluster gas pollution.

After studying the high-mass clusters, we began a systematic study of low-mass GCs, FG-only and mainly-FG GCs, and highmass and old open clusters (OCs). Our goal is to empirically find the mass limit for the appearance of the Na-O anti-correlation and to ascertain whether there are differences between high-mass and low-mass cluster properties, for instance in the relative fraction of FG and SG stars.

We also included GCs belonging to the Sagittarius dwarf spheroidal galaxy (Sgr dSph) to study whether there are differences in GCs formed in different environments (the MW and dwarf galaxies). GCs born in a dSph are expected to have experienced a milder tidal field, thus possibly retaining a larger portion of their original mass. Only a few old GCs in Fornax and LMC have their abundances derived using high-resolution spectroscopy. These GCs also seem to host two populations (Letarte et al. 2006; Johnson et al. 2006; Mucciarelli et al. 2009), but the fractions of FG and SG stars in Fornax and LMC GCs of similar mass seem different. Is this again a problem of low statistics, or is the galactic environment (a dwarf spheroidal vs. a dwarf irregular) influencing the GC formation mechanism? This is outside the main theme of this paper, and we refer to Larsen et al. (2012), D'Antona et al. (2013) for a more detailed discussion.

We have already gathered high-resolution spectra of a lowmass GC (NGC 6535, which will be analysed in a forthcoming paper), two high-mass OCs (Berkeley 39, NGC 6791, see Bragaglia et al. 2012, 2014), a proposed FG-only GC (NGC 6139, this paper), and Terzan 8 (Carretta et al. 2014a), which belongs to the Sgr dSph. In Terzan 8 we see some indication of a SG, at variance with other low-mass Sgr GCs (Ter 7, Pal 12, Tautvaišiené et al. 2004; Sbordone et al. 2007; Cohen 2004) or to Rup 106 (Villanova et al. 2013). However, in Terzan 8 the SG seems to be a minority component, in contrast to what occurs for high-mass GCs. The Na-O anti-correlation has never been observed in OCs (de Silva et al. 2009; MacLean et al. 2014), and we confirmed this using large samples of stars both for Berkeley 39 (Bragaglia et al. 2012) using FLAMES spectra and NGC 6791 (Bragaglia et al. 2014) using Hydra at WIYN and HIRES at Keck spectra. In particular, for NGC 6791, where Geisler et al. (2012) claimed to have found some variations in $\mathrm{Na}, \mathrm{O}$, we did not detect any evidence of this trend. Our findings are corroborated by independent works, see Cunha et al. (2015), Boesgaard et al. (2015).

We concentrate here on the possibly FG-only cluster NGC 6139. In Sect. 2 we present literature information on the cluster, and in Sect. 3 we describe the photometric data and the spectroscopic observations and derive the atmospheric

\footnotetext{
This appears to occur, for instance, for NGC 6397 and NGC 6838 (Carretta et al. 2009b), for which Gratton et al. (2010) found that the He dispersion is not necessary to explain their HBs. However, Milone et al. (2012b) studied the main sequence of NGC 6397 and found an internal variation in $\mathrm{Y}$ of about 0.01 , which is small, but exceeds their measurement error.
}

parameters. The abundance analysis is presented in Sect. 4, and we discuss the light-element abundances in Sect. 5.

\section{NGC 6139 in literature}

NGC 6139 is a massive GC (its absolute visual magnitude, a proxy for mass, is $M_{V}=-8.36$, Harris 1996) located toward the centre of the MW, at $l=342.37^{\circ}, b=6.94^{\circ}$. The cluster has received relatively little attention in the past; in particular, it has never before been studied with high-resolution spectroscopy.

Hazen (1991) found ten variable stars in NGC 6139, five of which are RR Lyrae stars. The four RRab seem to indicate a Oosterhoff type II. A CMD was presented by Samus' et al. (1996); they employed photographic $B$ and $V$ plates, and their photometry only reached the RGB and HB. They found the cluster to be quite metal-poor (close to $[\mathrm{Fe} / \mathrm{H}]=-2$ ) from the slope of the RGB, and highly reddened. Zinn \& Barnes (1998) used VI photometry that barely reached the main-sequence turnoff, noted the high and differential reddening (and corrected for a gradient), and determined $[\mathrm{Fe} / \mathrm{H}]=-1.71 \pm 0.20$ and $\mathrm{a}$ mean reddening of $E(V-I)=1.03 \pm 0.04$, corresponding to $E(B-V)=0.76 \pm 0.03$. Similar results were reached by Ortolani et al. (1999) on the basis of VI photometry, and by Davidge (1998), who used near-infrared data and also noted that the differential reddening is not a significant problem, since the RGB sequence becomes very well defined once the field star contamination is statistically removed. Finally, Piotto et al. (2002) presented WFPC2 photometry, as part of their HST snapshot programme (74 MW GCs observed with the $F 439 \mathrm{~W}$ and $F 555 \mathrm{~W}$ bands), in which the RGB and HB are very well defined, and the main-sequence turn-off is better defined than in previous works. Their data are publicly available, and we used them for the present work (see next section).

The only determination of metallicity based on spectroscopy is reported by Saviane et al. (2012), who used spectra at a resolution of $R \sim 2500$ in the region of the infrared Ca II triplet (CaT). NGC 6139 is one of the 20 GCs in the paper. They observed 19 stars, 15 of which were considered members (we used this information to select our targets, see next section). The metallicity they obtained from the CaT lines is $[\mathrm{Fe} / \mathrm{H}]=-1.63, \mathrm{rms}=$ 0.13 (on the metallicity scale defined in Carretta et al. 2009c).

Caloi \& D'Antona (2011) included NGC 6139 in their list of candidate FG clusters. They tried to identify clusters whose HB could be reproduced by a single mass (in the framework where the dispersion in mass of HB stars is due -at least in part- to variations in He content, in turn an indication of multiple generations). A first clue is the short $\mathrm{HB}$ (similar to the HB of NGC 6397, see Piotto et al. 2002, which, however has both a normal Na-O anti-correlation and a small $\Delta \mathrm{Y}$; see Carretta et al. 2009a,b; Milone et al. 2012b, respectively). However, given its high mass $\left(\log M=5.58 M_{\odot}\right.$, their Table 1), NGC 6139 should have produced a SG, but failed to lose a large part of the FG stars, so it should currently be FG-dominated. Caloi \& D'Antona (2011) did not discuss the case for NGC 6139 in detail, but because it was included in the list, combined with the absence of previous high-resolution spectroscopic studies, it was a good target for our on-going programme.

\section{Observations and analysis}

Of the photometric data discussed in the previous section, only the HST catalogue is publicly available. However, the very small field of view (FoV, about $2^{\prime}$ side) is poorly suited to the 


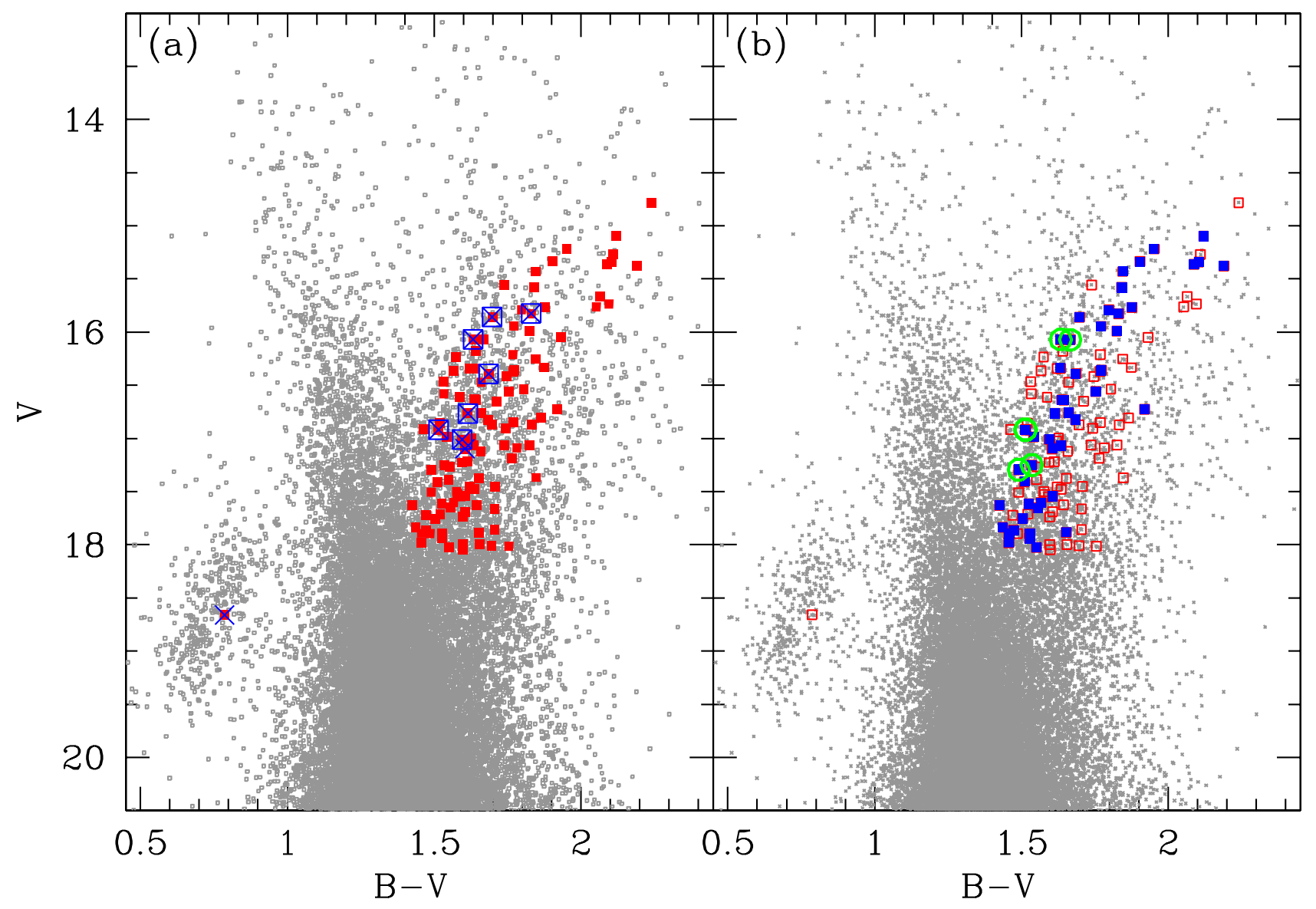

Fig. 1. a) CMD of NGC 6139, with its quite short blue HB. In grey we plot all stars, in red all targets observed with FLAMES. The seven UVES stars are indicated by blue open squares; the stars with RV from Saviane et al. (2012) are represented by blue crosses. b) The non-member stars (and the star on the HB) are indicated by red open squares, the stars that are candidate members on the basis of their RV by filled blue squares. The member stars define a reasonably tight RGB, and we indicate with green circles the probable AGB stars (see Sect. 3.1)

Table 1. Log of FLAMES observations.

\begin{tabular}{lccccc}
\hline \hline Setup & $\begin{array}{c}\text { UT date } \\
\text { (yyyy-mm-dd) }\end{array}$ & $\begin{array}{c}\mathrm{UT}_{\text {init }} \\
\text { (hh:mm:ss) }\end{array}$ & $\begin{array}{c}\text { Exptime } \\
\text { (s) }\end{array}$ & Airmass & $\begin{array}{c}\text { Seeing } \\
\text { (arcsec) }\end{array}$ \\
\hline HR11 & $2014-05-10$ & $02: 25: 58.027$ & 3600 & 1.459 & 0.76 \\
HR11 & $2014-05-10$ & $03: 27: 50.586$ & 3600 & 1.217 & 0.88 \\
HR11 & $2014-05-10$ & $04: 29: 58.656$ & 3600 & 1.091 & 0.64 \\
HR11 & $2014-05-10$ & $05: 36: 41.844$ & 3600 & 1.035 & 0.68 \\
HR11 & $2014-05-10$ & $06: 50: 54.214$ & 3600 & 1.052 & 0.57 \\
HR11 & $2014-05-10$ & $07: 54: 38.745$ & 3600 & 1.136 & 0.47 \\
HR13 & $2014-07-22$ & $03: 21: 00.913$ & 3600 & 1.164 & 1.06 \\
HR13 & $2014-07-22$ & $04: 24: 36.587$ & 3600 & 1.366 & 1.09 \\
HR13 & $2014-07-25$ & $03: 14: 57.248$ & 3600 & 1.178 & 0.76 \\
HR13 & $2014-07-26$ & $04: 12: 36.160$ & 3600 & 1.383 & 0.59 \\
HR21 & $2014-06-03$ & $01: 01: 28.004$ & 3090 & 1.409 & 0.86 \\
HR21 & $2014-07-03$ & $00: 29: 34.582$ & 3090 & 1.137 & 0.99 \\
\hline
\end{tabular}

FLAMES FoV (25' diameter). We therefore retrieved from the ESO archive some $B$ and $V$ filter frames acquired (under program 68.D-0265) with the Wide Field Imager (WFI) at the $2.2 \mathrm{~m}$ ESO-MPG telescope, which has a FoV of about $30^{\prime}$ side.

They were reduced in the standard way, correcting for bias and flat field using IRAF $^{2}$. Stars were detected independently

\footnotetext{
2 IRAF is distributed by the National Optical Astronomical Observatory, which are operated by the Association of Universities for Research in Astronomy, under contract with the National Science Foundation.
}

in the $B$ and $V$ frames, and the instrumental magnitudes were obtained using the point spread function (PSF) fitting code DAOPHOT-II/ALLSTAR (Stetson 1987, 1993). We employed the 2 Micron All Sky Survey Catalogue (2MASS, Skrutskie et al. 2006) and the CataXcorr code ${ }^{3}$, developed by P. Montegriffo, to compute the astrometric solution and transform the instrumental pixel coordinates into J2000 celestial coordinates. The astrometric precision is about 0.2 arcsec, perfectly compatible with the requirements of the FLAMES observations. No standard stars were available, so we calibrated our photometry to that of the HST using the stars in common. The final photometric catalogue will be made available through the CDS.

The resulting CMD is shown in Fig. 1. As expected from its Galactic position, the field star contamination is conspicuous, but the cluster RGB and HB are visible, especially in the very central region. The cluster sequences are also affected by differential reddening (DR), as already discussed in the literature. This could be relevant for the spectroscopic analysis, since our atmospheric parameters are derived from photometry. However, as has previously been done for other difficult cases, resorting to optical-IR colours (in particular, $V-K$ ), greatly alleviates the problem. For instance, the bulge GC GC 6441 (Gratton et al. 2007) has an rms scatter of $0.05 \mathrm{mag}$ in $E(B-V)$, resulting in random (star-to-star) uncertainties in the effective temperatures of $\pm 80 \mathrm{~K}$. We roughly evaluated the size of DR for NGC 6139 by defining an RGB ridge line with the help of HST photometry

http://www.bo.astro.it/ paolo/Main/CataPack.html 
and by projecting the candidate members (see next section) on it along the reddening vector. The average displacement required is $0.03 \mathrm{mag}$, therefore we expect a possible error of about $50 \mathrm{~K}$.

\subsection{FLAMES spectra}

NGC 6139 was observed with the multi-object spectrograph FLAMES at VLT (Pasquini et al. 2002). We used the GIRAFFE high-resolution setups HR11, HR13, and HR21 $(R=24200$, 22500,16200 , respectively), which contain two Na doublets, the [O I] line at $6300 \AA$, and the $\mathrm{Al}$ doublet at $8772 \AA$, plus several $\mathrm{Mg}$ lines. The observations were performed in service mode; a log is presented in Table 1. The HR11 and HR13 GIRAFFE observations were coupled with the high-resolution $(R=47000)$ UVES $580 \mathrm{~nm}$ setup $(\lambda \lambda \simeq 4800-6800 \AA)$ and the HR21 observations with the UVES $860 \mathrm{~nm}$ setup $(\lambda \lambda \simeq 6600-10600 \AA)$. We only used the $580 \mathrm{~nm}$ spectra in this paper for consistency with our previous works and because they have better signal-tonoise ratio.

The $B, V$ WFI catalogue was used to select candidate targets for the spectroscopic observations. As for all other GCs in our sample, we only chose stars without close neighbours $\left(\leq 2.5^{\prime \prime}\right)$. We cross-identified the stars in Saviane et al. (2012) to allocate seven UVES fibres to high-probability cluster members; the eigth fibre was put on an empty spot for sky subtraction. We allocated 103 GIRAFFE fibres to RGB stars, one to an $\mathrm{HB}$ star, and 16 to sky positions. All targets are brighter than the RGB bump ( $V=17.867 \pm 0.019$; Nataf et al. 2013). The 111 stars observed are shown in Fig. 1a with different symbols; their coordinates and magnitudes are given in Table A.1 (for candidate members, based on their RV) and Table A.2 (for non-members). All stars observed are within 7 arcmin from the centre, see Fig. 2, lower panel, that is, they are well within the tidal radius (10.5 armin, Harris 1996). Almost all stars observed are beyond the half-mass radius (0.85 arcmin, Harris).

The spectra were reduced using the ESO pipelines for UVES-FIBRE and GIRAFFE data; they take care of bias and flat-field correction, order tracing, extraction, fibre transmission, scattered light, and wavelength calibration. We then used IRAF routines on the $1 \mathrm{D}$ wavelength-calibrated individual spectra to subtract the (average) sky, shift to zero RV, and combine all the exposures for each star. The region near the [O I] line required special attention because of the strong sky emission and the many absorptions. Given the low RV of the cluster, the HR13 exposures were scheduled when the Earth motion took the sky emission farther away from the [O I] line of the stars. We also checked that in all these exposures the telluric absorptions did not fall on the line of the stars by visual comparison with a telluric template. The RV was measured using DOOp (Cantat-Gaudin et al. 2014), an automated wrapper for DAOSPEC (Stetson \& Pancino 2008); the average, heliocentric value for each star is given in Tables A.1 and A.2.

We show in Fig. 2 the histogram of the RVs and a plot of $\mathrm{RVs}$ versus distance from cluster centre (taken from the website update in 2010 of Harris 1996); the cluster signature is clear. We are the first to report an estimate of RV based on a large number of stars observed at high resolution; we found $\langle\mathrm{RV}\rangle=$ $+28.88, \mathrm{rms}=7.34 \mathrm{~km} \mathrm{~s}^{-1}$ (a typical rms for a GC). This value is compatible with the $\mathrm{RV}=6.7 \pm 6.0 \mathrm{~km} \mathrm{~s}^{-1}$ in Harris (1996), based on much lower resolution and lower quality data ${ }^{4}$, and it

\footnotetext{
4 Harris (1996) refers to Webbink (1981), who used RVs from Kinman (1959), derived on spectra at $R \sim 200$ of four stars.
}
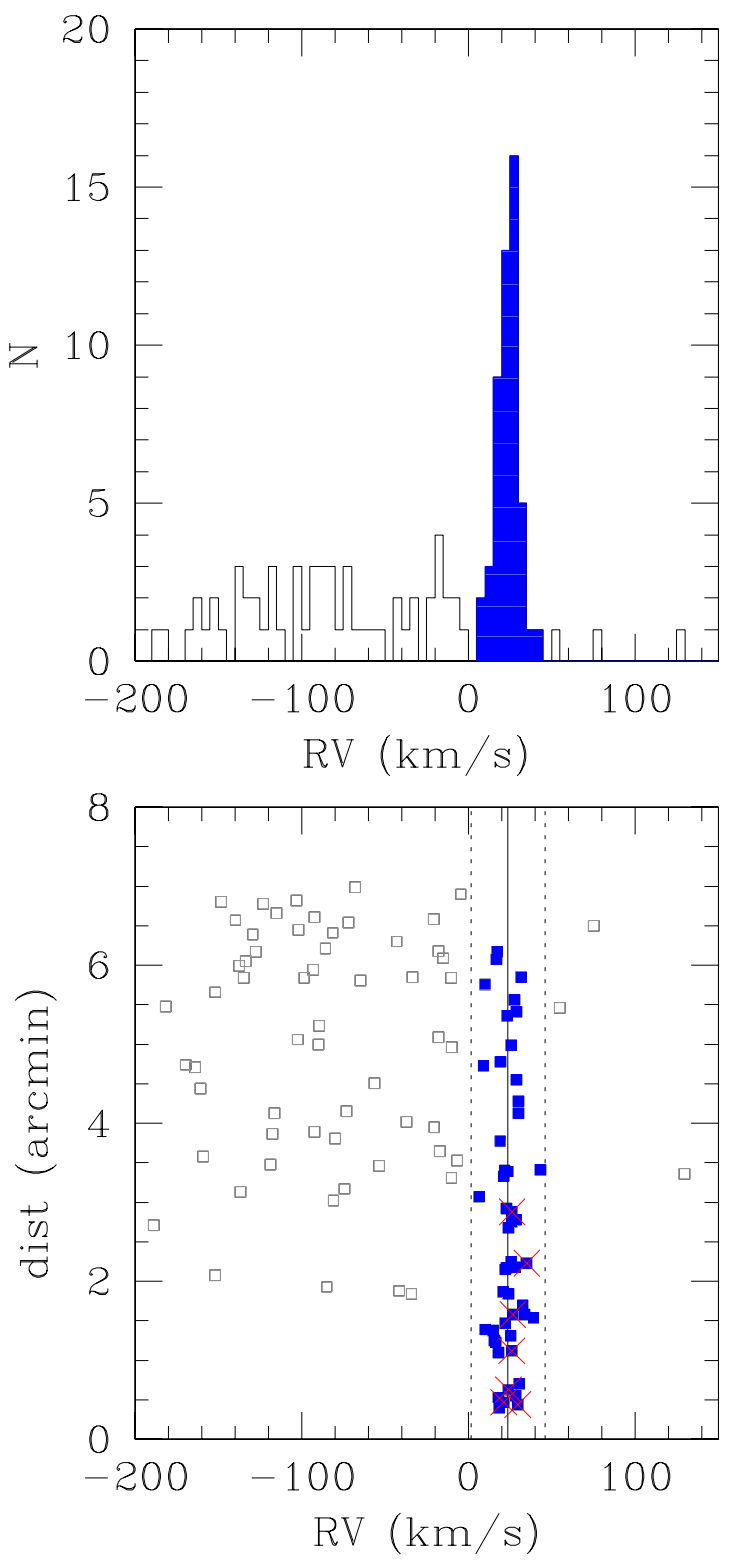

Fig. 2. Upper panel: histogram of all measured RVs (open histogram) and of the candidate member stars alone (blue filled histogram). Lower panel: RVs versus distance from the cluster centre. The candidate member stars are indicated by filled blue squares, the UVES targets by large red crosses, and the lines indicate the cluster mean RV and the $\pm 3 \sigma$ interval.

agrees well with Saviane et al. (2012), who reported a RV = $34 \pm 4 \mathrm{~km} \mathrm{~s}^{-1}$, based on 15 stars (for a direct comparison of RVs for eight stars in common, see Table A.1).

We are left with 50 candidate members based on the RV, that is, falling within $\pm 3 \sigma$ of the cluster average. After pruning the sample, the RGB of NGC 6139 is well defined and tight, even in presence of DR (see Fig. 1b). In the following we discuss only these 50 candidates.

Following our well-tested procedure (for a lengthy description, see e.g., Carretta et al. 2009a,b), effective temperatures $T_{\text {eff }}$ for our targets were derived using an average relation between apparent magnitudes and first-pass temperatures from $V-K$ colours and the calibrations of Alonso et al. (1999, 2001). This method permits decreasing the star-to-star errors in abundances due to uncertainties in temperatures, since magnitudes are less 
Table 2. Sensitivities of abundance ratios to variations in the atmospheric parameters and to errors in the equivalent widths, and errors in abundances for stars of NGC 6139 observed with UVES.

\begin{tabular}{lrrrrrrrr}
\hline \hline Element & $\begin{array}{r}\text { Average } \\
\text { n. lines }\end{array}$ & $\begin{array}{r}T_{\text {eff }} \\
(\mathrm{K})\end{array}$ & $\begin{array}{r}\log g \\
(\mathrm{dex})\end{array}$ & $\begin{array}{r}{[\mathrm{A} / \mathrm{H}]} \\
(\mathrm{dex})\end{array}$ & $\begin{array}{r}v_{t} \\
\mathrm{~km} \mathrm{~s}^{-1}\end{array}$ & $\begin{array}{c}E W \mathrm{~s} \\
(\mathrm{dex})\end{array}$ & $\begin{array}{c}\text { Total } \\
\text { internal }\end{array}$ & $\begin{array}{c}\text { Total } \\
\text { systematic }\end{array}$ \\
\hline Variation & & 50 & 0.20 & 0.10 & 0.10 & & & \\
Internal & & 4 & 0.04 & 0.04 & 0.06 & 0.01 & & \\
Systematic & & 46 & 0.06 & 0.06 & 0.02 & & & \\
\hline$[\mathrm{Fe} / \mathrm{H}] \mathrm{I}$ & 86 & +0.060 & -0.002 & -0.008 & -0.028 & 0.011 & 0.021 & 0.058 \\
{$[\mathrm{Fe} / \mathrm{H}] \mathrm{II}$} & 16 & -0.031 & +0.086 & +0.022 & -0.014 & 0.026 & 0.034 & 0.042 \\
{$[\mathrm{O} / \mathrm{Fe}] \mathrm{I}$} & 1 & -0.044 & +0.084 & +0.038 & +0.027 & 0.104 & 0.108 & 0.096 \\
{$[\mathrm{Na} / \mathrm{Fe}] \mathrm{I}$} & 2 & -0.016 & -0.038 & -0.015 & +0.022 & 0.074 & 0.075 & 0.088 \\
{$[\mathrm{Mg} / \mathrm{Fe}] \mathrm{I}$} & 2 & -0.016 & -0.009 & -0.000 & +0.012 & 0.074 & 0.074 & 0.021 \\
{$[\mathrm{Al} / \mathrm{Fe}] \mathrm{I}$} & 2 & -0.017 & -0.008 & -0.002 & +0.026 & 0.074 & 0.075 & 0.077 \\
\hline
\end{tabular}

affected by uncertainties than colours. This is particularly true for NGC 6139, which presents high and variable reddening, for which we used the apparent $K$ magnitudes in our relation with $T_{\text {eff }}$ because the effect of the DR on these magnitudes is weaker. The adopted reddening $E(B-V)=0.75$ and distance modulus $(m-M)_{V}=17.35$ are taken from the catalogue of Harris (1996). Gravities were obtained from apparent magnitudes and distance modulus, assuming the bolometric corrections from Alonso et al. (1999). We adopted a mass of $0.85 M_{\odot}$ for all stars and $M_{\mathrm{bol}, \odot}=4.75$ as the bolometric magnitude for the Sun, as in our previous studies.

We eliminated trends in the relation between abundances from Fe I lines and expected line strength (Magain 1984) to obtain values of the microturbulent velocity $v_{t}$.

Finally, using the above values, we interpolated within the Kurucz (1993) grid of model atmospheres (with the option for overshooting on) to derive the final abundances, adopting for each star the model with the appropriate atmospheric parameters and whose abundances matched those derived from Fe I lines. Five stars among the candidate members have a metallicity higher by more than 0.5 dex than the average of the others; they are probably field stars and were excluded from the most secure sample of RV plus metallicity members. The atmospheric parameters for all bona fide member stars are given in Table A.3.

Six of the candidate members (two with UVES and four with GIRAFFE spectra) were too metal-poor (by about $0.15 \mathrm{dex}$ ); their position in the CMD seemed to indicate that they might be asymptotic giant branch (AGB) and not RGB stars. Following the same procedure as was used for the RGB stars, we derived a separate colour-temperature relation for them, finding temperatures higher by about $110 \mathrm{~K}$. Adopting the new temperatures, we repeated the analysis, finding a metallicity that agreed very well with the cluster average. These second values for $T_{\text {eff }}$ and metallicity are given in Table A.3.

\section{Abundances}

In addition to the abundance of Fe, we present here the abundance of $\mathrm{O}, \mathrm{Na}, \mathrm{Mg}$, and $\mathrm{Al}$. The last was derived from the Al I doublet at 6696-98 $\AA$ for stars observed with UVES and from the doublet at $8772 \AA$ for stars observed with GIRAFFE. The abundance ratios for all elements are given in Table A.4, together with number of lines used and the rms scatter.

The abundances were derived using equivalent widths (EW). We measured the EW of iron and other elements using the code ROSA (Gratton 1988) adopting a relationship between EW and FWHM (for details, see Bragaglia et al. 2001). The atomic data for all the lines in the UVES spectra and in the HR11 and HR13 set-ups and the solar reference values come from Gratton et al. (2003). The Na abundances were corrected for departure form local thermodynamical equilibrium according to Gratton et al. (1999).

The derived Fe abundances do not show any trend with $T_{\text {eff }}$. The average metallicity derived for stars with UVES spectra is $[\mathrm{Fe} / \mathrm{H}]=-1.579 \pm 0.015 \pm 0.058(\mathrm{rms}=0.040 \mathrm{dex}, 7$ stars $)$ from neutral species, where the first error is from statistics and the second is systematic (see below for the computation). For stars with GIRAFFE spectra we found a very similar value: $[\mathrm{Fe} / \mathrm{H}]=-1.596 \pm 0.006 \pm 0.042(\mathrm{rms}=0.038 \mathrm{dex}, 38 \mathrm{stars})$. The abundance derived from single ionized species agrees very well. We found $[\mathrm{Fe} / \mathrm{H}] \mathrm{II}=-1.541 \pm 0.016 \pm 0.054(\mathrm{rms}=$ $0.043 \mathrm{dex}, 7$ stars $)$ and $[\mathrm{Fe} / \mathrm{H}] \mathrm{II}=-1.579 \pm 0.008 \pm 0.042(\mathrm{rms}=$ 0.048 dex, 38 stars) for UVES and GIRAFFE, respectively. This supports the adopted temperature scale and gravities.

We here report the first high-resolution spectroscopic study of this cluster, which means that no real comparison with previous determinations is possible. However, the metallicity we find agrees well with the one based on $\mathrm{CaT}([\mathrm{Fe} / \mathrm{H}]=-1.63$, Saviane et al. 2012) and with the general finding of a low metallicity ( -1.7 or -2$)$ based on photometry.

To estimate the error budget, we closely followed the procedure described in Carretta et al. (2009a,b). Table 2 (for UVES spectra) and Table 3 (for GIRAFFE spectra) provide the sensitivities of abundance ratios to errors in atmospheric parameters and EWs and the internal and systematic errors. We call systematic errors the errors that are different for the various GCs considered in our series and that produce scatter in relations involving different GCs; however, they do not affect the star-to-star scatter in NGC 6139. The cluster uncertainty in $T_{\text {eff }}$ can be estimated by multiplying the slope of the relation $T_{\text {eff }}-(V-K)$ in NGC 6139 and the uncertainty in $E(V-K)$ (assumed to be $0.055 \mathrm{mag}$ ). We also quadratically summed a contribution from a conservative estimate of $0.02 \mathrm{mag}$ error in the zero point of $V-K$ colour. The resulting uncertainty is propagated together with those in distance modulus and stellar mass to estimate the systematic uncertainty in surface gravity, while the systematic error in $v_{t}$ is simply obtained by dividing the internal error for the square root of the number of stars. The cluster (systematic) error in the metallicity for NGC 6139 is then obtained by the quadratic sum of the above three terms (multiplied for the proper sensitivity) with the statistical errors of individual abundance determination. The sensitivities were obtained by repeating the abundance analysis for all stars, while changing one atmospheric parameter at the time, then taking the average; this was done separately for UVES and GIRAFFE spectra. The amount of change in the 
Table 3. Sensitivities of abundance ratios to variations in the atmospheric parameters and to errors in the equivalent widths, and errors in abundances for stars of NGC 6139 observed with GIRAFFE.

\begin{tabular}{lrrrrrrrr}
\hline \hline Element & $\begin{array}{r}\text { Average } \\
\text { n. lines }\end{array}$ & $\begin{array}{r}T_{\text {eff }} \\
(\mathrm{K})\end{array}$ & $\begin{array}{r}\log g \\
(\mathrm{dex})\end{array}$ & $\begin{array}{r}{[\mathrm{A} / \mathrm{H}]} \\
(\mathrm{dex})\end{array}$ & $\begin{array}{r}v_{t} \\
\mathrm{~km} \mathrm{~s}^{-1}\end{array}$ & $\begin{array}{r}E W \mathrm{~s} \\
(\mathrm{dex})\end{array}$ & $\begin{array}{r}\text { Total } \\
\text { internal }\end{array}$ & $\begin{array}{r}\text { Total } \\
\text { systematic }\end{array}$ \\
\hline Variation & & 50 & 0.20 & 0.10 & 0.10 & & & \\
Internal & & 4 & 0.04 & 0.04 & 0.13 & 0.02 & & \\
Systematic & & 46 & 0.06 & 0.05 & 0.02 & & & \\
\hline$[\mathrm{Fe} / \mathrm{H}] \mathrm{I}$ & 35 & +0.058 & -0.001 & -0.007 & -0.025 & 0.022 & 0.041 & 0.054 \\
{$[\mathrm{Fe} / \mathrm{H}] \mathrm{II}$} & 2 & -0.035 & +0.088 & +0.020 & -0.007 & 0.067 & 0.101 & 0.042 \\
{$[\mathrm{O} / \mathrm{Fe}] \mathrm{I}$} & 1 & -0.041 & +0.081 & +0.034 & +0.023 & 0.095 & 0.144 & 0.053 \\
{$[\mathrm{Na} / \mathrm{Fe}] \mathrm{I}$} & 3 & -0.016 & -0.040 & -0.018 & +0.017 & 0.067 & 0.084 & 0.043 \\
{$[\mathrm{Mg} / \mathrm{Fe}] \mathrm{I}$} & 2 & -0.021 & -0.009 & -0.003 & +0.013 & 0.095 & 0.100 & 0.021 \\
{$[\mathrm{Al} / \mathrm{Fe}] \mathrm{I}$} & 2 & -0.030 & -0.006 & -0.002 & +0.019 & 0.095 & 0.101 & 0.051 \\
\hline
\end{tabular}

input parameters used in the sensitivity computations is given in the table header.

\section{Light element anti-correlations}

Our final sample consists of 45 giant stars (seven observed with UVES and 38 with GIRAFFE, no stars are in common). We were able to measure $\mathrm{Na}, \mathrm{Mg}$, and $\mathrm{Al}$ in all spectra (UVES and GIRAFFE), while $\mathrm{O}$ was measured in all seven UVES stars, but only in 36 GIRAFFE stars (29 actual detections and seven upper limits).

The resulting relations between $\mathrm{O}$ and $\mathrm{Na}, \mathrm{Mg}$ and $\mathrm{Al}$ are shown in Fig. 3, where we clearly see the normal pattern of light-element abundance ratios in GCs. NGC 6139 displays a Na-O anti-correlation like all massive GCs studied to date. Interestingly, it also shows strong variation in $\mathrm{Al}$ coupled with a moderate variation in $\mathrm{Mg}$. A variation in $\mathrm{Al}$ is not seen in all GCs (see Fig. 6 in Carretta et al. 2009b); however, also when $\mathrm{Al}$ varies the moderate variation in $\mathrm{Mg}$ is typical, with only rare exceptions such as NGC 2808 and NGC 6752 (Carretta 2014; Carretta et al. 2012). On the other hand, we observe a clear NaAl correlation, with a Pearson correlation coefficient $r_{\mathrm{P}}=0.70$, which is very significant. This confirms that the $\mathrm{Na}-\mathrm{Na}$ and $\mathrm{Mg}-$ Al cycles are related, but also suggests that they do not occur in exactly the same polluting stars.

Using the criteria defined in Carretta et al. (2009a), we can define the fraction of stars belonging to the primordial $(\mathrm{P})$ and second-generation (I, E) components. The percentage of firstgeneration $\mathrm{P}$ stars is $26 \% \pm 8 \%$; the percentage of secondgeneration I stars (with moderate modification of the abundances) is $74 \% \pm 13 \%$; finally, there are no second-generation E stars (with extremely modified composition) in our sample of 43 stars in NGC 6139. These numbers agree with what is found in all massive Milky Way GCs (see, e.g., Carretta et al. 2010a, 2014b; Johnson \& Pilachowski 2012, and references therein); SG stars are currently the dominant population in them. Our study then does not confirm NGC 6139 as a mainly-FG cluster, as proposed by Caloi \& D'Antona (2011).

As for the other GCs in our sample (e.g., Carretta et al. 2009a), for which most of the abundance ratios are based on GIRAFFE spectra, the errors on abundances produce almost continuous distributions (Fig. 3). However, a separation between the $\mathrm{P}$ and I stars is visible in the upper panel ( $\mathrm{Na}$ and $\mathrm{O}$ ), especially for the stars observed with UVES. A clear-cut separation in groups along the $\mathrm{Na}-\mathrm{O}$ anti correlation has only been seen in high-quality and high-resolution data, such as the UVES spectra in M4 (Marino et al. 2008) and NGC 2808 (Carretta 2014). The
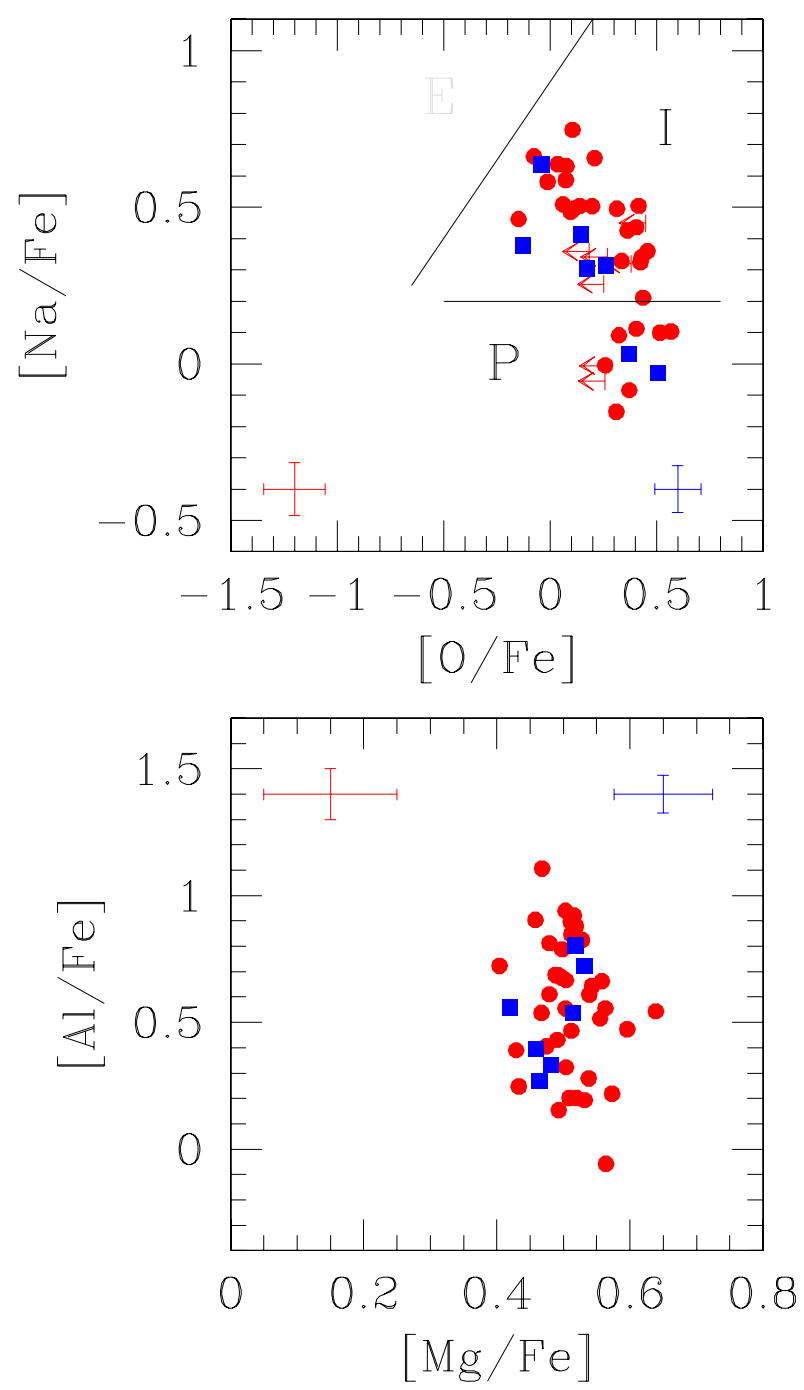

Fig. 3. Anti-correlations between light elements: Na-O (upper panel) and $\mathrm{Mg}$-Al (lower panel). GIRAFFE stars are indicated by red filled circles and UVES stars by filled blue squares; upper limits in $\mathrm{O}$ are shown as arrows. The error bars are the cluster average internal (starto-star) errors for each element for the GIRAFFE sample in red and UVES in blue (see text). In the upper panel we indicate the separation between $\mathrm{P}$ and I, and I and E populations, according to Carretta et al. (2009a).

Mg-Al distribution (Fig. 3 lower panel) looks continuous, with a total excursion of about 1 dex in $[\mathrm{Al} / \mathrm{Fe}]$. 


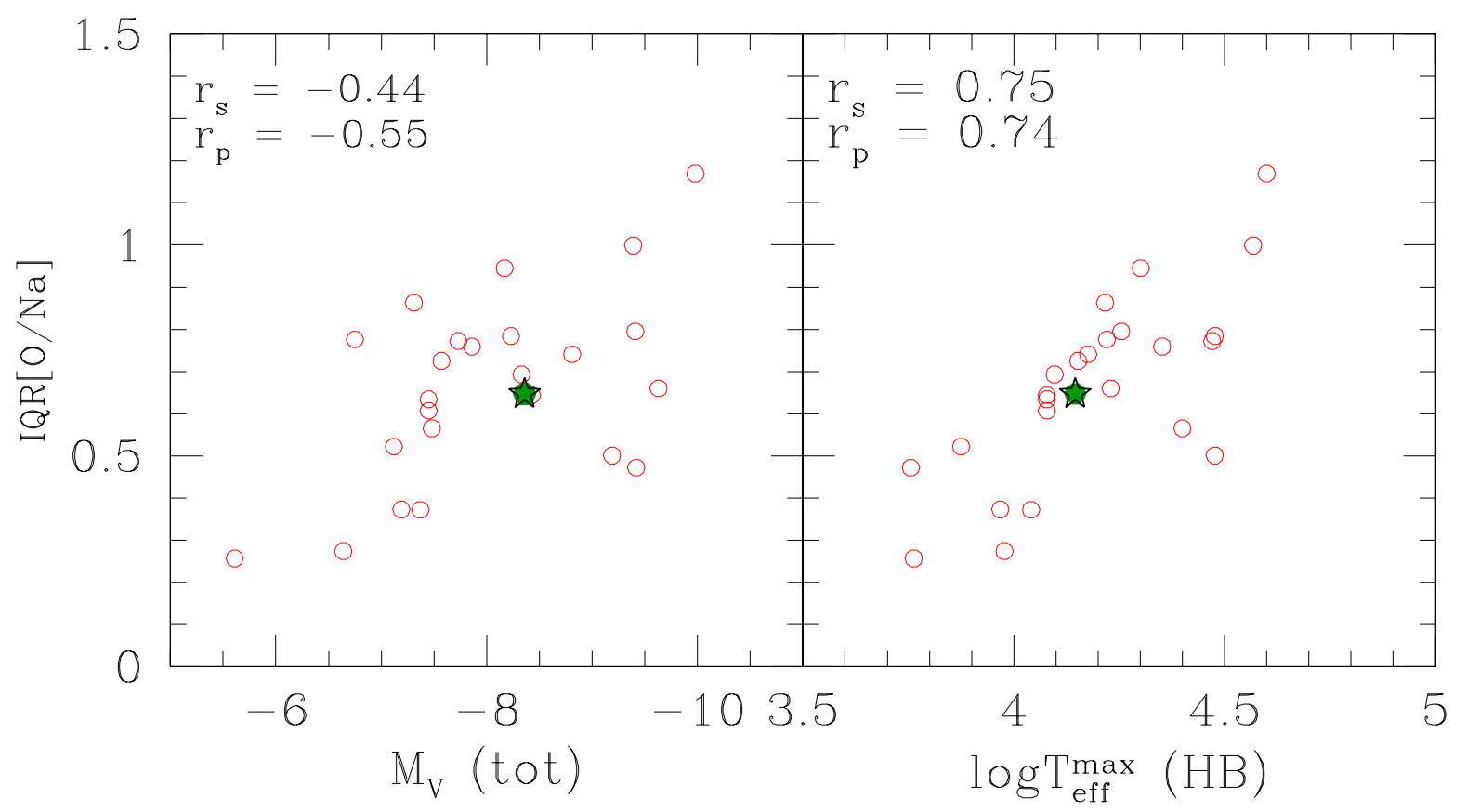

Fig. 4. Left panel: correlation between total absolute magnitude and the interquartile range of the [O/Na] ratio for GC in our FLAMES survey (Carretta et al. 2009a, 2010b, 2011a, 2013b, 2014b, 2015, and references therein). Right panel: relation between the maximum temperature along the HB (Recio-Blanco et al. 2006; Carretta et al. 2007) and IQR[O/Na]. In each panel NGC 6139 indicated by a filled green star. The Spearman rank correlation coefficient $r_{\mathrm{s}}$ and the Pearson linear correlation coefficient $r_{\mathrm{p}}$ are listed in each panel.

The Na-O anti-correlation has moderate extension, with an interquartile range (IQR) for the $[\mathrm{O} / \mathrm{Na}]$ ratio (Carretta 2006) $\mathrm{IQR}[\mathrm{O} / \mathrm{Na}]=0.647$. This IQR makes NGC 6139 fit in the relations with absolute magnitude $\left(M_{V}\right.$, i.e., mass) and maximum temperature reached on the HB $\left(T_{\mathrm{eff}}^{\max }(\mathrm{HB})\right.$, Recio-Blanco et al. 2006; Carretta et al. 2007, see Fig. 4 for a graphical representation).

In conclusion, with our study of 45 member stars in NGC 6139 conducted with FLAMES spectra, we determined for the first time its metallicity with high-resolution spectroscopy (the cluster has an intermediate metallicity, with $[\mathrm{Fe} / \mathrm{H}]=$ -1.58 dex). We also measured the light elements $\mathrm{O}, \mathrm{Na}, \mathrm{Mg}$, and $\mathrm{Al}$ involved in proton-capture reactions of $\mathrm{H}$-burning at high temperatures; we detected the usual correlations and anticorrelations found in almost all GCs investigated so far. The ratio of first-to-second generation stars in NGC 6139 is typical of the majority of GCs and is consistent with its relatively high total present-day mass. We do not support the idea that NGC 6139 is an FG-dominated cluster. The intermediate extension of the $\mathrm{Na}-\mathrm{O}$ anti-correlation, as measured by the interquartile range of the $[\mathrm{O} / \mathrm{Na}]$ ratio, fits the relation with the maximum temperature along the HB found by Carretta et al. (2007) very well. All in all, NGC 6139 behaves like a normal MW GC of similar mass and $\mathrm{HB}$ extension. The formation event that shaped its internal chemistry seems consistent with the pattern of a regular, relatively high-mass blue HB GC.

However, the finding of strong variation in $\mathrm{Al}$, which should also imply some spread in $\mathrm{He}$, does not perfectly agree with the $\mathrm{HB}$ of the cluster. We calculated IQR $[\mathrm{Al} / \mathrm{Mg}]$, finding a value of 0.425; according to Fig. 28 in Gratton et al. (2010); this would require a $\Delta Y$ of about 0.02 (but note that the relation between $\mathrm{IQR}[\mathrm{Al} / \mathrm{Mg}]$ and $\Delta Y$ is rather poorly determined, being based on only eight points). We note also that the more massive, metal-poorer NGC 5024 (M53, with $M_{V}=-8.71$, $[\mathrm{Fe} / \mathrm{H}]=-2.10$, Harris 1996), proposed to be an FG-dominated
GC by Caloi \& D'Antona (2011) on the basis of its HB and discussed at length in that paper, has been demonstrated to host a substantial SG by Mészáros et al. (2015), using APOGEE data. NGC 5024 has a wide spread in $\mathrm{Al}$ (with a bimodal distribution) and almost no $\mathrm{Mg}$ variation; it behaves like NGC 5272 (M3, $M_{V}=-8.88,[\mathrm{Fe} / \mathrm{H}]=-1.50$, Harris 1996$)$, another $\mathrm{GC}$ with a rather short $\mathrm{HB}$, even more massive than NGC 6139, but with a similar metallicity. The relation between $\mathrm{Al}$ and $\mathrm{He}$ is evidently not completely understood. As as an additional cautionary note, Bastian et al. (2015) claimed that none of the enrichment mechanisms proposed to explain multiple populations in GCs can consistently do so, because all predict too wide He spreads to reproduce the observed spreads in light elements, notably those in $\mathrm{Na}$ and $\mathrm{O}$. More observational constraints are required, both from spectroscopy with large samples of stars for which elements of all nucleosynthetic chains are measured (as we and other groups are doing for an increasing number of GCs) and from precise photometry (e.g., Piotto et al. 2015, to determine He). These constraints will be helpful in improving the modelling of the formation and early enrichment phases of GCs.

Acknowledgements. We thank the referee for constructive comments that improved the paper. This research has made use of WEBDA, SIMBAD database, operated at CDS, Strasbourg, France, and NASA's Astrophysical Data System. This publication makes use of data products from the Two Micron All Sky Survey, which is a joint project of the University of Massachusetts and the Infrared Processing and Analysis Center/California Institute of Technology, funded by the National Aeronautics and Space Administration and the National Science Foundation. This research has been partially funded by PRIN INAF 2011 "Multiple populations in globular clusters: their role in the Galaxy assembly", and PRIN MIUR 2010-2011, project "The Chemical and Dynamical Evolution of the Milky Way and Local Group Galaxies".

\section{References}

Alonso, A., Arribas, S., \& Martínez-Roger, C. 1999, A\&AS, 140, 261 Alonso, A., Arribas, S., \& Martínez-Roger, C. 2001, A\&A, 376, 1039 
Bastian, N., Lamers, H. J. G. L. M., de Mink, S. E., et al. 2013, MNRAS, 436, 2398

Bastian, N., Cabrera-Ziri, I., \& Salaris, M. 2015, MNRAS, 449, 3333

Boesgaard, A. M., Lum, M. G., \& Deliyannis, C. P. 2015, ApJ, 799, 202

Bragaglia, A., Carretta, E., Gratton, R. G., et al. 2001, AJ, 121, 327

Bragaglia, A., Gratton, R. G., Carretta, E., et al. 2012, A\&A, 548, A122

Bragaglia, A., Sneden, C., Carretta, E., et al. 2014, ApJ, 796, 68

Caloi, V., \& D'Antona, F. 2011, MNRAS, 417, 228

Cantat-Gaudin, T., Donati, P., Pancino, E., et al. 2014, A\&A, 562, A10

Carretta, E. 2006, AJ, 131, 1766

Carretta, E. 2014, ApJ, 795, L28

Carretta, E., Recio-Blanco, A., Gratton, R. G., Piotto, G., \& Bragaglia, A. 2007, ApJ, 671, L125

Carretta, E., Bragaglia, A., Gratton, R. G., et al. 2009a, A\&A, 505, 117

Carretta, E., Bragaglia, A., Gratton, R. G., \& Lucatello, S. 2009b, A\&A, 505, 139

Carretta, E., Bragaglia, A., Gratton, R. G., D’Orazi, V., \& Lucatello, S. 2009c, A\&A, 508, 695

Carretta, E., Bragaglia, A., Gratton, R. G., et al. 2010a, A\&A, 516, A55

Carretta, E., Bragaglia, A., Gratton, R. G., et al. 2010b, A\&A, 520, A95

Carretta, E., Lucatello, S., Gratton, R. G., Bragaglia, A., \& D'Orazi, V. 2011a, A\&A, 533, A69

Carretta, E., Bragaglia, A., Gratton, R., D’Orazi, V., \& Lucatello, S. 2011b, A\&A, 535, A121

Carretta, E., Bragaglia, A., Gratton, R. G., Lucatello, S., \& D’Orazi, V. 2012, ApJ, 750, L14

Carretta, E., Gratton, R. G., Bragaglia, A., D’Orazi, V., \& Lucatello, S. 2013a, A\&A, 550, A34

Carretta, E., Bragaglia, A., Gratton, R. G., et al. 2013b, A\&A, 557, A138

Carretta, E., Bragaglia, A., Gratton, R. G., et al. 2014a, A\&A, 561, A87

Carretta, E., Bragaglia, A., Gratton, R. G., et al. 2014b, A\&A, 564, A60

Carretta, E., Bragaglia, A., Gratton, R. G., et al. 2015, A\&A, 578, A116

Cohen, J. G. 2004, AJ, 127, 1545

Cunha, K., Smith, V. V., Johnson, J. A., et al. 2015, ApJ, 798, L41

D’Antona, F., Bellazzini, M., Caloi, V., et al. 2005, ApJ, 631, 868

D'Antona, F., Caloi, V., D'Ercole, A., et al. 2013, MNRAS, 434, 1138

Davidge, T. J. 1998, AJ, 116, 1744

Decressin, T., Meynet, G., Charbonnel, C., Prantzos, N., \& Ekström, S. 2007, A\&A, 464, 1029

Geisler, D., Villanova, S., Carraro, G., et al. 2012, ApJ, 756, L40

de Silva, G. M., Gibson, B. K., Lattanzio, J., \& Asplund, M. 2009, A\&A, 500, L25

Gratton, R. G. 1988, Rome Obs. Preprint Ser., 29

Gratton, R. G., Carretta, E., Eriksson, K., \& Gustafsson, B. 1999, A\&A, 350, 955

Gratton, R. G., Carretta, E., Claudi, R., Lucatello, S., \& Barbieri, M. 2003, A\&A, 404, 187

Gratton, R., Sneden, C., \& Carretta, E. 2004, ARA\&A, 42, 385

Gratton, R. G., Lucatello, S., Bragaglia, A., et al. 2007, A\&A, 464, 953
Gratton, R. G., Carretta, E., Bragaglia, A., Lucatello, S., \& D’Orazi, V. 2010, A\&A, 517, A81

Gratton, R. G., Carretta, E., \& Bragaglia, A. 2012, A\&ARv, 20, 50

Harris, W. E. 1996, AJ, 112, 1487

Hazen, M. L. 1991, AJ, 101, 170

Johnson, C. I., \& Pilachowski, C. A. 2012, ApJ, 754, L38

Johnson, J. A., Ivans, I. I., \& Stetson, P. B. 2006, ApJ, 640, 801

Kinman, T. D. 1959 , MNRAS, 119,157

Kurucz, R. 1993, ATLAS9 Stellar Atmosphere Programs and $2 \mathrm{~km} \mathrm{~s}^{-1}$ grid, CD-ROM No. 13 (Cambridge, Mass.: Smithsonian Astrophysical Observatory)

Larsen, S. S., Strader, J., \& Brodie, J. P. 2012, A\&A, 544, L14

Letarte, B., Hill, V., Jablonka, P., et al. 2006, A\&A, 453, 547

MacLean, B. T., De Silva, G. M., \& Lattanzio, J. 2014, MNRAS, 466, 3556

Magain, P. 1984, A\&A, 134, 189

Marino, A. F., Villanova, S., Piotto, G., et al. 2008, A\&A, 490, 625

Mészáros, S., Martell, S. L., Shetrone, M., et al. 2015, AJ, 149, 153

Milone, A. P., Piotto, G., Bedin, L. R., et al. 2012a, ApJ, 744, 58

Milone, A. P., Marino, A. F., Piotto, G., et al. 2012b, ApJ, 745, 27

Milone, A. P., Marino, A. F., Dotter, A., et al. 2014, ApJ, 785, 21

Mucciarelli, A., Origlia, L., Ferraro, F. R., \& Pancino, E. 2009, ApJ, 695, L134

Nataf, D. M., Gould, A. P., Pinsonneault, M. H., \& Udalski, A. 2013, ApJ, 766, 77

Ortolani, S., Bica, E., \& Barbuy, B. 1999, A\&AS, 138, 267

Pasquini, L., Avila, G., Blecha, A., et al. 2002, The Messenger, 110, 1

Piotto, G., King, I. R., Djorgovski, S. G., et al. 2002, A\&A, 391, 945

Piotto, G., Bedin, L. R., Anderson, J., et al. 2007, ApJ, 661, L53

Piotto, G., Milone, A. P., Bedin, L. R., et al. 2015, AJ, 149, 91

Prantzos, N., Charbonnel, C., \& Iliadis, C. 2007, A\&A, 470, 179

Recio-Blanco, A., Aparicio, A., Piotto, G., de Angeli, F., \& Djorgovski, S. G. 2006, A\&A, 452, 875

Samus', N. N., Kravtsov, V. V., Pavlov, M. V., et al. 1996, Astron. Lett., 22, 686

Saviane, I., da Costa, G. S., Held, E. V., et al. 2012, A\&A, 540, A27

Sbordone, L., Bonifacio, P., Buonanno, R., et al. 2007, A\&A, 465, 815

Sbordone, L., Salaris, M., Weiss, A., \& Cassisi, S. 2011, A\&A, 534, A9

Skrutskie, M. F., Cutri, R. M., Stiening, R., et al. 2006, AJ, 131, 1163

Stetson, P. B. 1987, PASP, 99, 191

Stetson, P. B. 1993, in Stellar photometry - Current techniques and future developments, eds. C. J. Butler, \& I. Elliott (Cambridge: Cambridge Univ. Press), Proc. IAU Colloq., 136, 291

Stetson, P. B., \& Pancino, E. 2008, PASP, 120, 1332

Tautvaišiene, G., Wallerstein, G., Geisler, D., Gonzalez, G., \& Charbonnel, C. 2004, AJ, 127, 373

Ventura, P., D'Antona, F., Mazzitelli, I., \& Gratton, R. 2001, ApJ, 550, L65

Villanova, S., Geisler, D., Carraro, G., Moni Bidin, C., \& Muñoz, C. 2013, ApJ, 778,186

Webbink, R. F. 1981, ApJS, 45, 259

Zinn, R., \& Barnes, S. 1998, AJ, 116, 1736 


\section{Appendix A: Additional tables}

Table A.1. Information on the member stars observed.

\begin{tabular}{|c|c|c|c|c|c|c|c|c|}
\hline ID & $\begin{array}{c}\text { RA } \\
\text { (hh:mm:ss) }\end{array}$ & $\begin{array}{c}\text { Dec } \\
\text { (dd:pp:ss) }\end{array}$ & $V$ & $B$ & $\begin{array}{c}K \\
\text { (2MASS) } \\
\end{array}$ & $\begin{array}{r}\mathrm{RV} \\
\left(\mathrm{km} \mathrm{s}^{-1}\right) \\
\end{array}$ & $\begin{array}{c}\begin{array}{c}\text { Err } \\
\left(\mathrm{km} \mathrm{s}^{-1}\right)\end{array} \\
\end{array}$ & Notes \\
\hline \multicolumn{9}{|c|}{ UVES } \\
\hline t00540 & $16: 27: 39.82$ & $-38: 50: 18.05$ & 15.825 & 17.656 & 10.601 & 24.00 & 0.30 & $4, \mathrm{RV}_{S 12}=37$ \\
\hline t00553 & $16: 27: 39.22$ & $-38: 51: 18.04$ & 15.859 & 17.557 & 10.856 & 29.63 & 0.60 & $1, \mathrm{RV}_{S 12}=46$ \\
\hline t00670 & $16: 27: 42.77$ & $-38: 50: 56.29$ & 16.069 & 17.703 & 11.329 & 21.27 & 0.50 & $3, \mathrm{RV}_{S 12}=36, \mathrm{AGB}$ \\
\hline t00951 & $16: 27: 35.86$ & $-38: 53: 40.28$ & 16.391 & 18.077 & 11.706 & 26.21 & 0.60 & $13, \mathrm{RV}_{S 12}=44$ \\
\hline t01348 & $16: 27: 40.82$ & $-38: 48: 42.10$ & 16.764 & 18.379 & 12.049 & 35.00 & 0.30 & $11, \mathrm{RV}_{S 12}=19$ \\
\hline t01554 & $16: 27: 38.41$ & $-38: 49: 23.44$ & 16.920 & 18.434 & 12.261 & 26.71 & 1.60 & $8, \mathrm{RV}_{S 12}=38, \mathrm{AGB}$ \\
\hline t01699 & $16: 27: 42.55$ & $-38: 49: 53.35$ & 17.010 & 18.605 & 12.383 & 26.09 & 0.50 & $7, \mathrm{RV}_{S 12}=42$ \\
\hline \multicolumn{9}{|c|}{ GIRAFFE, members } \\
\hline t00261 & $16: 27: 43.32$ & $-38: 57: 04.20$ & 15.097 & 17.217 & 9.530 & 17.37 & 0.35 & \\
\hline t00284 & $16: 27: 46.06$ & $-38: 54: 03.74$ & 15.218 & 17.170 & 9.988 & 21.40 & 0.55 & \\
\hline
\end{tabular}

Notes. In the notes we provide the number and RV in Saviane et al. (2012); Star t06169 is on the HB. The full table is available at the CDS.

Table A.2. Information on non-member stars on the basis of RV or metallicity, all observed with GIRAFFE.

\begin{tabular}{lcccccccc}
\hline \hline ID & $\begin{array}{c}\text { RA } \\
\text { (hh:mm:ss })\end{array}$ & $\begin{array}{c}\text { Dec } \\
\text { (dd:pp:ss) }\end{array}$ & $V$ & $B$ & $\begin{array}{c}K \\
(2 \mathrm{MASS})\end{array}$ & $\begin{array}{c}\mathrm{RV} \\
\left(\mathrm{km} \mathrm{s}^{-1}\right)\end{array}$ & $\begin{array}{c}\text { Err } \\
\left(\mathrm{km} \mathrm{s}^{-1}\right)\end{array}$ & Note \\
\hline t00187 & $16: 27: 25.45$ & $-38: 48: 42.66$ & 14.781 & 17.020 & 7.078 & -16.98 & 1.00 & $\mathrm{RV}$ \\
t00306 & $16: 27: 10.97$ & $-38: 53: 19.81$ & 15.271 & 17.381 & 9.388 & -85.72 & 0.49 & $\mathrm{RV}$ \\
t00392 & $16: 27: 48.91$ & $-38: 51: 43.38$ & 15.555 & 17.294 & 10.639 & -34.11 & 0.99 & $\mathrm{RV}$ \\
t00466 & $16: 28: 07.92$ & $-38: 49: 47.99$ & 15.666 & 17.730 & 9.262 & -181.62 & 0.69 & $\mathrm{RV}$ \\
t00513 & $16: 28: 00.26$ & $-38: 52: 21.00$ & 15.737 & 17.832 & 9.739 & -116.30 & 0.48 & $\mathrm{RV}$ \\
$\ldots$ & & & & & & & & \\
\hline
\end{tabular}

Notes. The full table is available at the CDS.

Table A.3. Adopted atmospheric parameters and derived metallicity for confirmed member stars.

\begin{tabular}{|c|c|c|c|c|c|c|c|c|c|c|}
\hline Star & $\begin{array}{l}T_{\text {eff }} \\
(\mathrm{K}) \\
\end{array}$ & $\begin{array}{l}\log g \\
(\operatorname{dex})\end{array}$ & $\begin{array}{c}{[\mathrm{A} / \mathrm{H}]} \\
(\mathrm{dex})\end{array}$ & $\begin{array}{c}\begin{array}{c}v_{t} \\
\left(\mathrm{~km} \mathrm{~s}^{-1}\right)\end{array} \\
\end{array}$ & $\mathrm{nr}$ & $\begin{array}{c}{[\mathrm{Fe} / \mathrm{H}] \mathrm{I}} \\
(\mathrm{dex})\end{array}$ & $\mathrm{rms}$ & $\mathrm{nr}$ & $\begin{array}{r}{[\mathrm{Fe} / \mathrm{HIII}} \\
(\mathrm{dex}) \\
\end{array}$ & rms \\
\hline \multicolumn{11}{|c|}{ UVES } \\
\hline t00540 & 4156 & 0.95 & -1.55 & 1.85 & 121 & -1.545 & 0.089 & 20 & -1.551 & 0.076 \\
\hline t00553 & 4212 & 1.07 & -1.61 & 1.40 & 107 & -1.610 & 0.102 & 20 & -1.505 & 0.131 \\
\hline t00670 & 4417 & 1.28 & -1.57 & 1.43 & 91 & -1.570 & 0.112 & 13 & -1.566 & 0.164 \\
\hline t00951 & 4398 & 1.44 & -1.61 & 1.93 & 83 & -1.614 & 0.095 & 17 & -1.475 & 0.098 \\
\hline t01348 & 4473 & 1.57 & -1.57 & 1.52 & 74 & -1.571 & 0.101 & 15 & -1.586 & 0.109 \\
\hline t01554 & 4634 & 1.76 & -1.63 & 0.98 & 52 & -1.629 & 0.140 & 10 & -1.588 & 0.173 \\
\hline \multirow{2}{*}{\multicolumn{11}{|c|}{ GIRAFFE }} \\
\hline & & & & & & & & & & \\
\hline t00261 & 3922 & 0.51 & -1.56 & 2.09 & 40 & -1.558 & 0.087 & 3 & -1.524 & 0.207 \\
\hline t00284 & 4022 & 0.71 & -1.59 & 1.97 & 36 & -1.592 & 0.099 & 3 & -1.517 & 0.064 \\
\hline
\end{tabular}

Notes. The full table is available at the CDS.

Table A.4. Light-element abundances for confirmed member stars.

\begin{tabular}{|c|c|c|c|c|c|c|c|c|c|c|c|c|c|c|}
\hline Star & $\mathrm{nr}$ & {$[\mathrm{O} / \mathrm{Fe}] \mathrm{I}$} & $\mathrm{rms}$ & $\mathrm{nr}$ & {$[\mathrm{Na} / \mathrm{Fe}] \mathrm{I}$} & $\mathrm{rms}$ & $\mathrm{nr}$ & {$[\mathrm{Mg} / \mathrm{Fe}] \mathrm{I}$} & $\mathrm{rms}$ & $\mathrm{nr}$ & {$[\mathrm{Al} / \mathrm{Fe}] \mathrm{I}$} & $\mathrm{rms}$ & Lim? & PIE \\
\hline \multicolumn{15}{|c|}{ UVES } \\
\hline t00540 & 1 & -0.041 & & 3 & 0.637 & 0.010 & 3 & 0.518 & 0.068 & 2 & 0.802 & 0.008 & & I \\
\hline t00553 & 2 & 0.174 & 0.071 & 3 & 0.303 & 0.050 & 3 & 0.459 & 0.038 & 2 & 0.395 & 0.084 & & I \\
\hline t00670 & 1 & -0.126 & & 2 & 0.377 & 0.089 & 2 & 0.420 & 0.071 & 2 & 0.559 & 0.006 & & I \\
\hline t00951 & 1 & 0.506 & & 1 & -0.028 & & 2 & 0.482 & 0.011 & 2 & 0.330 & 0.126 & & $\mathrm{P}$ \\
\hline t01348 & 1 & 0.369 & & 2 & 0.030 & 0.055 & 2 & 0.464 & 0.006 & 1 & 0.270 & & & $\mathrm{P}$ \\
\hline t01554 & 1 & 0.263 & & 2 & 0.313 & 0.061 & 2 & 0.532 & 0.204 & 2 & 0.724 & 0.097 & & I \\
\hline t01699 & 1 & 0.146 & & 2 & 0.414 & 0.001 & 2 & 0.514 & 0.102 & 2 & 0.536 & 0.112 & & I \\
\hline \multicolumn{15}{|c|}{ GIRAFFE } \\
\hline t00284 & 2 & 0.209 & 0.006 & 4 & 0.657 & 0.040 & 3 & 0.563 & 0.057 & 2 & 0.556 & 0.000 & & I \\
\hline
\end{tabular}

Notes. The full table is available at the CDS. 